\title{
Decentralized Data Dissemination and Harvesting for Urban Monitoring
}

\author{
M. Milojevic and J. A. Barria \\ Electrical and Electronic Engineering Department \\ Imperial College London, UK \\ Emails: \{m.milojevic13, j.barria\}@imperial.ac.uk
}

\begin{abstract}
This paper presents a Decentralized data Dissemination and Harvesting (DDH) mechanism for urban pollution monitoring using mobile sensor nodes with limited resources. The proposed DDH mechanism enables participating nodes to self-decide whether to process the received data or not, thus, reducing the on-board processing load. Based on the harvested data, the nodes calculate their level of interest in monitoring the particular street segments. In this way a reduction in the number of actively participating nodes is accomplished. In addition, the mobile nodes process raw sensor readings using the Delayed State Information Filter (DSIF) to maintain the past pollution states and perform a decentralized data fusion. The proposed DDH mechanism is assessed using simulations with varying number of the participating nodes. The results show that the proposed mechanism outperforms existing solutions in terms of the utilisation of nodes resources, without affecting the amount of volume of gathered data for the monitored street segments.
\end{abstract}

Keywords: Vehicular sensor network, data dissemination, data harvesting, decentralized monitoring

\section{INTRODUCTION}

One of the biggest problems facing urban public authorities, due to the pace of urbanization and growth of vehicular activity, is high air pollution levels. Having a high spatiotemporal resolution of pollution data would enable authorities to, e.g., issue early warnings at the onset of the harmful level of pollution episodes.

At present, air pollution monitoring is usually done via fixed monitoring stations or Wireless Sensor Networks (WSNs) [1]. Such approach provides a low spatial resolution of pollution data referring to the entire or large parts of the city [2]. Estimating the pollution data with high spatial resolution at the level of street segments remains a challenge. This is because covering every street segment with a fixed sensing units would require considerable investment. Alternatively, urban sensing can be done via Vehicular Sensor Network (VSN) consisting of vehicles (e.g. cars, buses, bicycles) equipped with the on-board units (sensors, database, processor, battery, GPS and 802.11p transceiver). In this way, monitoring locations that otherwise could not be covered with fixed sensors could be reached using the existing urban transportation network. Vehicles collect the pollution data along the routes and communicate it to other vehicles in the network or servers that perform data analysis or visualization [3].
The use of public bicycles in everyday transportation is a growing trend in the large cities and it could be used for implementing VSN sensing concept. For example, an environmental sensing project using bicycles in Copenhagen is presented in [4]. The main restriction of using bicycles for environmental sensing is that, unlike cars or buses [5]-[8], they have limited resources such as database, processing capability and battery power. Taking into account aforementioned constraints, we present a Decentralized data Dissemination and Harvesting (DDH) mechanism designed for urban sensing applications (e.g. pollution monitoring) using the mobile nodes with limited resources (e.g. public bicycles). Distributed sensing also raises a question of how to deal with the vast amount of data obtained from diverse sources (sensors) [9]. To address this, the DDH's mobile nodes have embedded the Delayed State Information Filter (DSIF) to refine sensor's readings and keep track of the evolution of the air pollution level. The goal of the proposed mechanism is to keep the communication and processing load at a minimum level without affecting the monitoring time span of the street segments. Moreover, the DDH mechanism provides the information that reduces the number of nodes monitoring the same street segments while nodes are moving in a non-controlled manner. The proposed algorithm is evaluated through an extensive set of simulations under a varying number of the participating nodes in the network. The results show that with significantly reduced resources, the DDH mechanism provides the same amount of gathered data across the urban area, as other existing approaches which do not consider the limited on-board resources.

The remainder of this paper is organized as follows: Section II describes related work, Section III presents the proposed mechanism. Section IV gives description of the simulation setup and experimental results. Section $\mathrm{V}$ outlines the final remarks.

\section{RELATED WORK}

Data gathering and dissemination in sensing applications has been thoroughly researched in WSNs. However, the architecture and characteristics of WSNs significantly differ from VSNs [3]. In WSNs nodes are hierarchically organized, with limited or no mobility at all and have central processing at data sink. With recent developments of Vehicular Ad Hoc Networks (VANETs), the concept of urban monitoring raised an interest in broad classes of applications and represents an 
emerging network paradigm [2]. Some of the vehicular sensing proposals can be found in [10] and survey of dissemination and opportunistic data gathering protocols is presented in [11] and [12], respectively.

The aim of applications in VANETs is to inform as many vehicles as possible about some event, for example accident or traffic congestion [13]. In addition, the vehicles in VANETs disseminate data frequently, which can cause spreading of the redundant information through the network. In case a node is not interested in disseminated information, receiving a huge amount of such data could lead to the unnecessary utilisation of its resources. Therefore, VANETs proposals for dissemination are not suitable for urban monitoring using nodes with the limited resources.

Authors in [6] present a pollution monitoring solution using the public transportation network where dissemination and harvesting is done in an opportunistic manner. Here buses equipped with sensors record raw sensor measurements on their route and share the collected data with other buses they encounter. A concept of mobile sensing and distributed opportunistic search of sensed data for proactive urban monitoring is presented in [9]. Mobile nodes perform sensing, periodically generate and disseminate summaries. Mobile agents roam inside a network and opportunistically harvest disseminated data from the mobile nodes. In [14] authors introduced the To-and-Fro (TaF) scheme, a localized data dissemination solution using opportunistic VSN. It is based on forwarding the message only to the vehicles moving in the opposite direction. Apart from urban sensing proposals, the well known proposal for monitoring wildlife realm is presented in [15]. Here two dissemination protocols are defined. The first one is simple flooding where every mobile node transmits all buffered data to its neighbors until base station receives it. The second one is a history-based protocol where the forwarder node is chosen as the one with the highest probability to encounter the base station.

Works such as [6] and [9] assume that the mobile nodes are not limited in terms of processing, power and database supply. Contrary to that, the proposed monitoring solution takes into account these constraints. It is also important to note that in our solution nodes do not transmit and store raw sensor measurements like in [6]. Instead, refining of the sensor readings is done using a filter. This enables extraction of the meaningful information from the noisy sensor observations taken by the devices of the different accuracy level. Mobility wise, [6] assumes controlled mobility and fixed routes which is unsuitable for achieving fine grained monitoring of the large areas. Contrary to that, our proposal is based on non-controlled mobility of the nodes. Thus, it enables more flexible movement which can result in higher spatial granularity of the collected data.

From the perspective of communication, flooding technique used in [15] can generate high communication load. Further, a history-based proposal is not applicable in realistic scenarios due to nodes' unpredictable movement. Unlike [9], where two types of vehicles are required to preform harvesting and dis- semination, in our mechanism these two features are merged and performed by every node. To accomplish harvesting task, the communication in [9] is query based which contributes to the increase of communication load. In addition, the mobile nodes broadcast their collected summaries and the mobile agents send their queries periodically every three seconds. As opposed to [9], our proposal is based on a single hop broadcasting when nodes enter the intersection area. The main downside of [14] is not considering the content of the messages. Hence, the nodes can potentially discard some relevant information originating from the non opposite direction.

\section{Decentralized Dissemination And HaRVESting (DDH) MECHANISM}

The proposed decentralized sensing mechanism relies on the existing infrastructure (e.g. public bicycles). It does not require any investment into dedicated nodes that would be used solely for monitoring purposes. We assume that nodes are equipped with a GPS and a digital map of a city in which roads are divided into street segments. One street segment is defined between two consecutive intersections and has a unique identification (ID) $j$, where $j=\{1, \ldots, S\}$ and $S$ is the total number of street segments in the city. A node periodically samples the level of Nitrogen Oxide (NO) pollution with a sampling period $T_{s}$. Subsequently, the measurements are processed using the Delayed State Information Filter (DSIF). It is the special form of the Information Filter that enables storing past states and is often used in decentralized systems [16]. Keeping the past states affects the information matrix and vector to grow over time. However, the structure of information matrix maintains block tridiagonal and sparse which does not deviate in time.

A state that is estimated $\left(x_{t}\right)$ is composed of pollution concentration value $(c)$ and speed of its change $(v)$ :

$$
x_{t}=\left(\begin{array}{ll}
c & v
\end{array}\right)^{T}
$$

The equations of the observed $\left(x_{t}\right)$ and measurement $\left(z_{t}\right)$ systems are defined as follows:

$$
x_{t}=F_{t} x_{t-1}+q_{t}, z_{t}=H_{t} x_{t}+r_{t}
$$

The measurement model is defined as $H_{t}=[10]$ and the motion model $\left(F_{t}\right)$ is represented as a discrete version of continuous white-noise acceleration model [17]:

$$
F_{t}=\left(\begin{array}{cc}
1 & T_{s} \\
0 & 1
\end{array}\right)
$$

The measurement noise $\left(r_{t} \sim N\left(0, R_{t}\right)\right)$ is assumed to be Gaussian white noise with zero mean and variance $R_{t}=\sigma_{r}^{2}$, where $\sigma_{r}$ is a standard deviation. The process noise $\left(q_{t} \sim\right.$ $N\left(0, Q_{t}\right)$ ) is assumed to be Gaussian white noise with zero mean, covariance $Q_{t}$, and standard deviation $\sigma_{q}$ :

$$
Q_{t}=\left(\begin{array}{cc}
\frac{T_{s}^{3}}{3} & \frac{T_{s}^{2}}{2} \\
\frac{T_{s}^{2}}{2} & T_{s}
\end{array}\right) \sigma_{q}^{2}
$$


The prediction step of the information matrix $\left(\bar{Y}_{t}\right)$ and the information state vector $\left(\bar{y}_{t}\right)$ for time instance $t$ are calculated as:

$$
\begin{gathered}
\bar{Y}_{t}=\left(\begin{array}{cc}
Q_{t}^{-1} & -Q_{t}^{-1} F_{t} \\
-F_{t}^{T} Q_{t}^{-1} & \hat{Y}_{t-1}+F_{t}^{T} Q_{t}^{-1} F_{t}
\end{array}\right) \\
\bar{y}_{t}=\left(\begin{array}{c}
0 \\
\hat{y}_{t-1}
\end{array}\right)
\end{gathered}
$$

When a measurement $\left(z_{t}\right)$ is received, the update step for time $t$ is performed, and the information matrix $\left(\hat{Y}_{t}\right)$ and information vector $\left(\hat{y}_{t}\right)$ are defined as:

$$
\begin{gathered}
\hat{Y}_{t}=\bar{Y}_{t}+\left(\begin{array}{cc}
H_{t}^{T} R_{t}^{-1} H_{t} & 0 \\
0 & 0
\end{array}\right) \\
\hat{y}_{t}=\bar{y}_{t}+\left(\begin{array}{c}
H_{t}^{T} R_{t}^{-1} z_{t} \\
0
\end{array}\right)
\end{gathered}
$$

Finally, the updated information vector and matrix are converted into an estimated state $\hat{x}_{t}=\hat{P}_{t} \hat{y}_{t}$ and its covariance $\hat{P}_{t}=\hat{Y}_{t}^{-1}$.

Now, suppose that node $i$ enters the street segment $j$ when the first estimate is produced in a time $t_{\text {init }}$. The estimates are created while node travels through $j$ and the last estimate is created in time $t_{\text {last }}$. Based on the DSIF equations, node $i$ forms a trajectory $\left(\tau_{j}\right)$ for a street segment $j$ which consists of a set of the state estimates $\tau_{j}=\left\{\hat{x}_{j}\left(t_{\text {init }}\right), \ldots, \hat{x}_{j}\left(t_{\text {last }}\right)\right\}$. In addition, for every $\tau_{j}$ we define trajectory length $\left(l_{j}\right)$ as a time interval between time instances $t_{\text {init }}$ and $t_{\text {last }}$. As a consequence of the opportunistic nature of the proposed communication in the network, a node can collect more trajectories for the same street segment in its database. Thus, a set of gathered trajectories is defined as $\Omega_{j i}=\left\{\tau_{j_{1}}, \tau_{j_{2}}, \ldots, \tau_{j_{K}}\right\}$, where $K$ is the number of collected trajectories. Fig. 1 shows an example set of two gathered trajectories for street segment $j$. When node $i$ traverses the street segment it marks its identification as the one that is passed. Additionally, node $i$ calculates two more parameters that are needed for the operation of the DDH mechanism. The first one is called proximity $\left(\delta_{j i}\right)$ and describes the current positioning of node $i$ relative to the street segment $j$ :

$$
\delta_{j i}=\left\{\begin{array}{l}
0, \text { if node } i \text { is moving away from } j \\
1, \text { if node } i \text { is approaching to the } j
\end{array}\right.
$$

The second parameter is current coverage $\left(\alpha_{j i}\right)$ which represents a union of all trajectory lengths for a street segment $j:$

$$
\alpha_{j i}=\bigcup_{k=1}^{K} l_{j_{k}}
$$

Parameters $j, \Omega_{j i}, \delta_{j i}$ and $\alpha_{j i}$ are stored in the node's database for every street segment. $L_{i}$ is the number of street segments for which node $i$ has the data stored. Writing into the database is done dynamically as the node moves and obtains or receives data from other nodes in the network.

In the DDH mechanism, node $i$ broadcasts the message upon exiting the street segment in the first upcoming intersection. This ensures the highest communication range due to

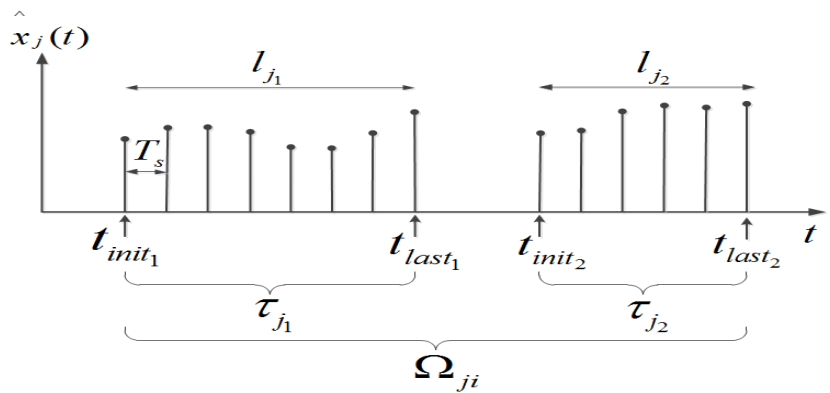

Fig. 1: A set of collected trajectories $\left(\Omega_{j i}\right)$ in the node's $i$ database for the street segment $j$.

least amount of surrounding obstacles. The formal structure of the message $(M)$ contains two parts. The first part $\left(M_{1}\right)$ is the set of trajectories of the last traversed street segment $\left(\Omega_{j_{\text {last }} i}\right)$ with its identification $\left(j_{\text {last }}\right)$. The second part $\left(M_{2}\right)$ contains the set of tuples of street segment identification, proximity and current coverage, that are defined for each stored street segment $\left(j_{1}, j_{2}, \ldots, j_{L_{i}}\right)$ :

$$
\begin{aligned}
M & =\left\{M_{1} ; M_{2}\right\} \\
& =\left\{\left(j_{\text {last }}, \Omega_{j_{\text {last }} i}\right) ;\left(j_{1}, \delta_{j_{1}}, \alpha_{j_{1} i}\right), \ldots,\left(j_{L_{i}}, \delta_{j_{L_{i}}}, \alpha_{j_{L_{i}} i} i\right)\right\}
\end{aligned}
$$

Its main role is to provide an insight into sensing performance of other nodes and enable the node to derive its level of interest in monitoring the street segments. The basic idea of harvesting mechanism is to provide a node the ability to self-decide if it should continue or stop monitoring a certain street segment. This mechanism is described by the pseudo-code presented in Algorithm 1.

Suppose the node $k$ harvests the message sent by the node $i$. The receiving node takes the street segment identification $j_{\text {last }}$ from the first part of the message $M_{1}$. It then calculates $\delta_{j_{\text {last }} k}$ parameter for that street segment and if it is 0 , the node $k$ discards $\Omega_{j_{\text {last }} i}$. Otherwise, it harvests $\Omega_{j_{\text {last }} i}$ and if there is already $\Omega_{j_{\text {last }} k}$ existing in a database, data fusion is performed based on trajectory synchronization [16]. Further, the node $k$ takes the second part of the message $M_{2}$ and compares it with its database. If the street segment ID $(j)$ match is found in the database and $M_{2}$ then the other two parameters proximity and current coverage are observed. In case the proximity parameters $\delta_{j i}$ and $\delta_{j k}$ are equal to 1 , the receiving node is interested in further analysis. For that particular street segment, the node $k$ compares its current coverage parameter with the harvested one. A node is not interested in further monitoring and deletes the stored data if its current coverage is lower than the one it received from the node $i$. In this way the receiving node $k$ interprets that some other node, in this case the node $i$, collected more data about the same street segment, and it is not necessary to keep it in its database. The node $k$ continues analyzing its database by checking the proximity parameters only of the non-traversed street segments. It is not interested in keeping the data about the street segments from which it is moving away, when the proximity is 0 . On the other hand, if the parameter is equal to 


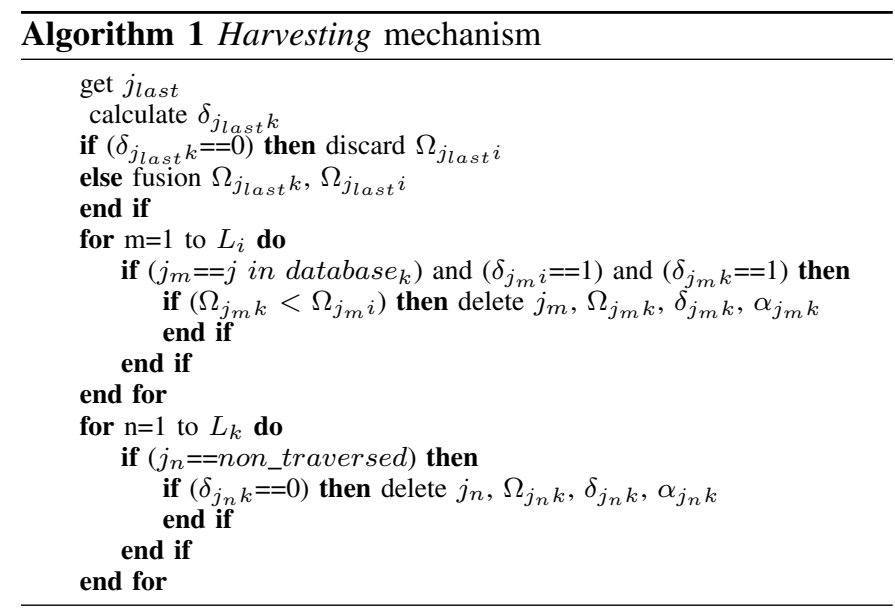

1 , node decides to keep the data since there is a possibility that it will enter that street segment in the future. Therefore, the stored data for which the node is not interested to keep further is considered to be irrelevant. This procedure of self-deciding and database maintenance enables reduction of the duplicated data propagating through the network.

\section{Simulation EVAluation}

\section{A. Simulation setup}

The DDH mechanism was evaluated via a set of simulations in an urban scenario performed in Veins [18] simulator. Veins bi-directionally couples the network simulator OMNET++ [19] and the road traffic simulator SUMO [20]. The urban environment in the simulation is based on Manhattan grid road network of $4 \mathrm{~km}^{2}$ size, $2 \times 2 \mathrm{~km}$ in dimensions. A network consists of 64 blocks with 144 street segments where each street segment is 250 meters long. The simulation includes a traffic light system and building blocks to make the radio propagation environment as realistic as possible. An example of a street segment scenario is presented in Fig. 2.

We performed three sets of simulations with different number of nodes in the network, 50, 100 and 200. Each node has a randomly created origin-destination route obtained by the shortest path Dijkstra algorithm. The arrival of the mobile nodes is simulated as a Poisson process with mean interarrival time of 3 seconds. The mobility of the nodes (bicycles) on the street segment is simulated according to Stefan Kraus model, as in [21]. In order to match the real bicycle specifications, parameters such as dimensions, acceleration, deceleration and minimum gap between the moving nodes are adjusted as in [20]. We set the node's maximum speed to $2 \mathrm{~m} / \mathrm{s}$, its transmitting power to $10 \mathrm{~mW}$, and the communication standard used is IEEE 802.11p [22]. The sampling period of embedded DSIF is set to 10 seconds with the process noise variance set to 0.6. One group of nodes has the measurement noise variance set to 1 and the other has measurement noise variance set to 1.2. The NO pollution data used in simulation is provided by the authors of [8] and the simulation time is set to 30 minutes. The results represent the average of 20 simulation runs within $95 \%$ confidence interval, calculated as in [23].
The proposed DDH mechanism is compared with adapted diffusion protocol from Mobeyes, where a node transmits a message periodically, containing the data about two street segments. Also, the DDH mechanism is compared with TaF where nodes broadcast a message during the fixed time interval containing the data about the last traversed street segment.

\section{B. Performance metrics}

To evaluate the performance of the DDH mechanism, we define the following metrics:

1) Processing load $\left(P_{L}\right)$ is defined as the average number of trajectories processed per node during its time spent in the simulation. This metric reflects node's processing activities and it should be kept as low as possible. It is calculated according to:

$$
P_{L}=\frac{\sum_{i} \sum_{j} \tau_{j i}}{N}
$$

where $i=\{1, \ldots, N\}$, number of nodes in the simulation $N=50,100,200$. A street segment identification $j=$ $\{1, \ldots, S\}$, where a total number of street segments in the city is $S=144$ and $\tau_{j i}$ represents processed trajectory for the street segment $j$ per node $i$.

2) Dissemination redundancy $\left(D_{R}\right)$ represents the average number of duplicated (redundant) trajectories received by the node referring to the same street segment for the same time interval. This metric shows the quantity of redundant information propagated through the network and the goal is to maintain its value to a minimum:

$$
D_{R}=\frac{\sum_{i} \sum_{j} \mu_{j i}}{N}
$$

where $\mu_{j i}$ represents the redundant trajectory per node $i$ for a street segment $j$.

3) Maximum coverage $\left(M_{C}\right)$ is defined as the average value of the maximum current coverage per street segment obtained by any single node. This metric allows us to evaluate a decentralized collection of pollution data and should be as high as possible:

$$
M_{C}=\frac{\sum_{j}\left(\alpha_{j i}\right)_{\max \mid i \in\{1, \ldots, N\}}}{S}
$$

where $\left(\alpha_{j i}\right)_{\max }$ is the maximum current coverage of the street segment $j$ obtained by node $i$.

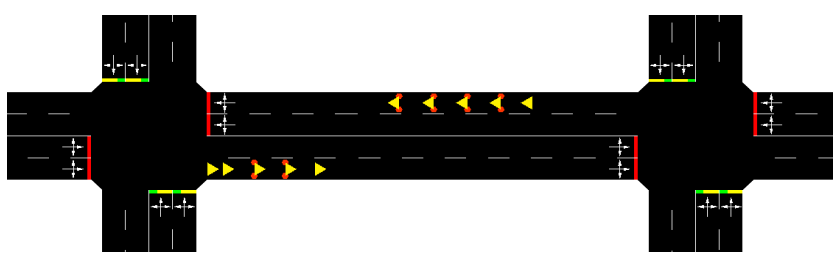

Fig. 2: An example of a street segment scenario from the SUMO simulator. 
4) Nodes participation in monitoring $\left(N_{P}\right)$ is defined as the average ratio of a total coverage of the street segment and the number of participating nodes. Merging the coverages gathered by the participating nodes represents a total coverage. The participating nodes are the ones that collected the data about the street segments, and this number should be as low as possible in order to enable effective node utilization. Thus, the metric's value should be high and is calculated according to:

$$
N_{P}=\frac{\sum_{j}\left(C_{j} / N_{j}\right)}{S}=\frac{\sum_{j}\left(\bigcup_{i} \alpha_{j i} / N_{j}\right)}{S}
$$

Here $C_{j}$ is the total coverage obtained for the street segment $j$ and $N_{j}$ is the number of participating nodes for that street segment.

5) Total coverage $\left(T_{C}\right)$ is defined as the average value of a total coverage per street segment. It shows how much time the street segment has been monitored and it should be as high as possible:

$$
T_{C}=\frac{\sum_{j} C_{j}}{S}
$$

\section{Simulation results}

The performance evaluation of the processing load and dissemination redundancy is depicted in Fig. 3 and Fig. 4, respectively. For both metrics the results show that the largest processing load and dissemination redundancy in all three cases is induced by Mobeyes diffusion and it is followed by TaF. The DDH mechanism outperforms Mobeyes and TaF achieving the lowest $P_{L}$ and $D_{R}$. The DDH's mechanism better performance is due to the fact that nodes consider the content of the received message prior to processing the data, and retain only the data that is in the interest for the urban monitoring. For example, the nodes do not process

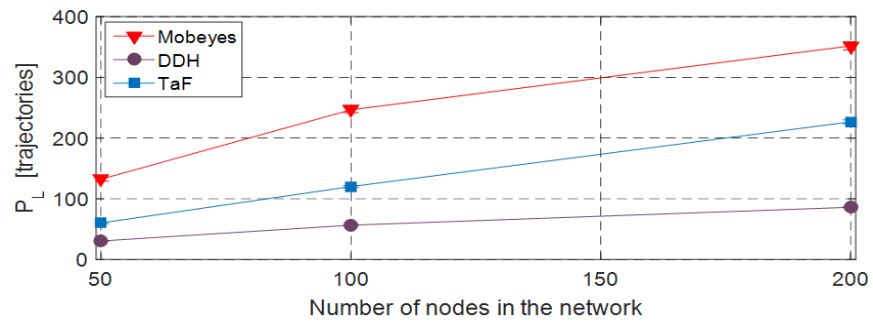

Fig. 3: Processing load $\left(P_{L}\right)$ evaluation.

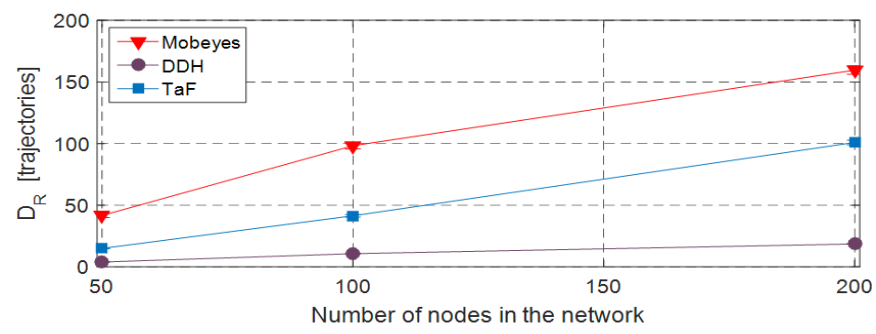

Fig. 4: Dissemination redundancy $\left(D_{R}\right)$ evaluation. the data if they are moving away from the street segment. Also, as a consequence of harvesting mechanism, the nodes check the content of the database in search for irrelevant data which is then deleted. Evaluation of $P_{L}$ shows that the DDH mechanism requires less data to be processed which is favourable in a case of nodes with limited resources.

Fig. 5 depicts the DDH mechanism performance in terms of the $M_{C}$ metric. As expected, Mobeyes achieves the highest $M_{C}$ due to the volume of data disseminated. In contrast, the DDH mechanism deletes irrelevant content from the node's database and significantly decreases $P_{L}$ and $D_{R}$. Therefore, the reduction of $P_{L}$ and $D_{R}$ by the DDH mechanism comes at the expense of a slight reduction of $M_{C}$. Even though TaF has more frequent broadcasting activity than the DDH mechanism, it produces lower $M_{C}$. This can be expected as TaF discards the data coming from the nodes moving in the same direction.

Results of the $N_{P}$ metric for different number of participating nodes in the network are shown in Fig. 6. They show that the DDH mechanism outperforms Mobeyes and TaF mostly due to its data harvesting characteristic which reduces the number of nodes carrying the same information. Increased number of nodes that monitor the same street segment produces unnecessary nodes utilization and causes spreading of the redundant data through the network.

The $T_{C}$ metric is shown in Fig. 7 and it can be seen that the DDH's mechanism policy of deleting selected stored data does not reduce the volume of gathered data monitored per street segments. This means that with the DDH mechanism, it is possible to achieve the same total coverage per street segment as Mobeyes and $\mathrm{TaF}$, however with less participating nodes.

Utilisation of the node's database is evaluated by comparing the average value of the total lengths of collected trajectories, as depicted in Fig. 8. From this figure it can be seen that the DDH mechanism database consumption is similar for 50 and 100 nodes, and it increases by $50 \%$ when the number of nodes

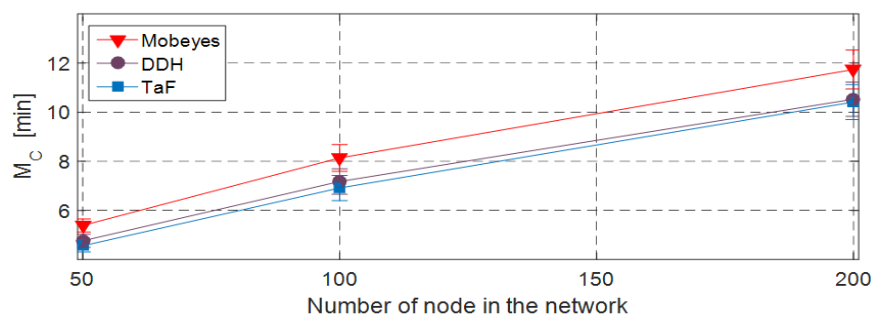

Fig. 5: Maximum coverage $\left(M_{C}\right)$ evaluation.

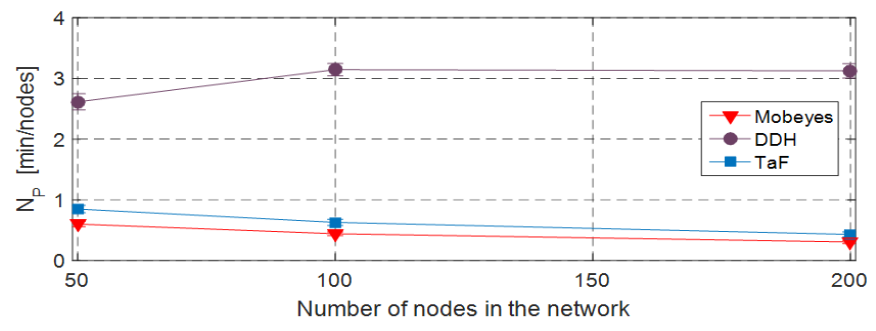

Fig. 6: Evaluation of nodes participation in monitoring $\left(N_{P}\right)$. 


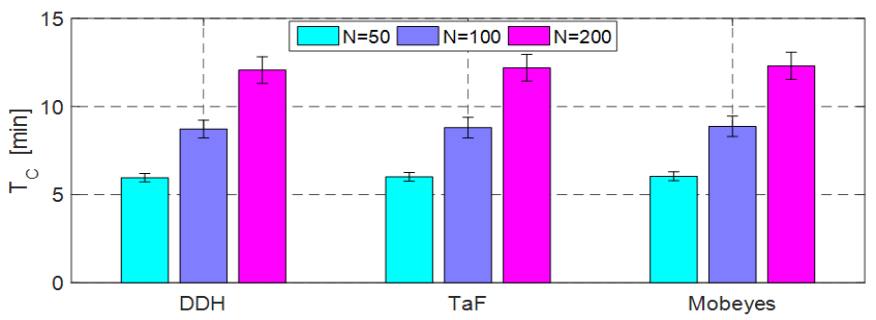

Fig. 7: Evaluation of the total coverage $\left(T_{C}\right)$.

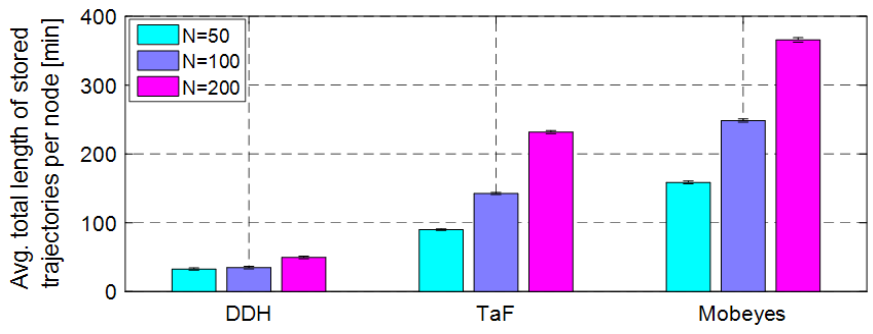

Fig. 8: Utilisation of node's database.

increases from 50 to 200. In contrast, in the case of Mobeyes and $\mathrm{TaF}$, this increase is larger (of the order of $\sim 150 \%$ ).

According to [9], we investigate the scalability of the DDH mechanism by observing the different metrics as we increase the number of nodes in the network. First, from Fig. 3 and Fig. 4 the $P_{L}$ and $D_{R}$ metrics do not significantly increase when the number of nodes augments from 50 up to 200. Also, Fig. 6 shows that the $N_{P}$ metrics remains almost unchanged when there is a higher number of the nodes in the network. Similarly, as seen in Fig. 8 the increase in database requirement is very low for the DDH mechanism when compared with Mobeyes and $\mathrm{TaF}$.

\section{FinAL REMARKS}

In this paper we present a Decentralized data Dissemination and Harvesting (DDH) mechanism for urban monitoring, which is based on the mobile sensor nodes with the limited resources. The nodes use the Delayed State Information Filter to refine raw sensor readings and perform data fusion in order to collect the state estimates from other nodes. Simulation results show that the DDH mechanism reduces the nodes' on-board processing load and the number of nodes required to monitor the street segments. The DDH mechanism also maintains low utilisation of the on-board node's database.

\section{REFERENCES}

[1] J. H. Liu, Y. F. Chen, T. S. Lin, C. P. Chen, P. T. Chen, T. H. Wen, C. H. Sun, J. Y. Juang and J. A. Jiang, "An air quality monitoring system for urban areas based on the technology of wireless sensor networks", International Journal on Smart Sensing and Intelligent Systems 5.1: 191214, 2012.

[2] B. Hull, V. Bychkovksy, Y. Zhang, K. Chen, M. Goraczko, A. Miu, E. Shin, H. Balakrishnan and S. Madden, "CarTel: a distributed mobile sensor computing system", In Proceedings of the 4th international conference on Embedded networked sensor systems, ACM, 125-138, 2006.

[3] B. Bahidja, "The sensors networks wsn and vsn: a theoretical comparison", International Journal of Wireless \& Mobile Networks 6.5: 59, 2014.
[4] C. Outram, C. Ratti, A. Biderman, "The Copenhagen Wheel: An innovative electric bicycle system that harnesses the power of realtime information and crowd sourcing", In EVER Monaco International Exhibition \& Conference on Ecologic Vehicles \& Renewable Energies, 2010.

[5] S. C. Hu, Y. C. Wang, C. Y. Huang and Y. C. Tseng, "Measuring air quality in city areas by vehicular wireless sensor networks", Journal of Systems and Software, 84.11, 2005-2012, 2011.

[6] F. Gil-Castineira, F. J. Gonzalez-Castano, R. J. Duro and F. LopezPena, "Urban pollution monitoring through opportunistic mobile sensor networks based on public transport", Computational Intelligence for Measurement Systems and Applications, 2008. CIMSA 2008. 2008 IEEE International Conference on. IEEE, 2008, 70-74, 2008.

[7] S. Devarakonda, P. Sevusu, H. Liu, R. Liu, L. Iftode and B. Nath, ”Realtime air quality monitoring through mobile sensing in metropolitan areas", In Proceedings of the 2nd ACM SIGKDD International Workshop on Urban Computing ACM, 2013.

[8] Y. Ma, M. Richards, M. Ghanem, Y. Guo and J. Hassard, "Air pollution monitoring and mining based on sensor grid in London", Sensors, 8.6: 3601-3623, 2008.

[9] U. Lee, E. Magistretti, M. Gerla, P. Bellavista and A. Corradi, 'Dissemination and harvesting of urban data using vehicular sensing platforms", Vehicular Technology, IEEE Transactions on, 58.2: 882-901, 2009.

[10] U. Lee and M. Gerla, "A survey of urban vehicular sensing platforms", Computer Networks, 54.4: 527-544, 2010.

[11] R. Daher, "Roadside Networks for Vehicular Communications: Architectures, Applications, and Test Fields: Architectures, Applications, and Test Fields", IGI Global, 2012.

[12] L. Pelusi, A. Passarella and M. Conti, "Opportunistic networking: data forwarding in disconnected mobile ad hoc networks", Communications Magazine, IEEE, 44.11: 134-141, 2006.

[13] S. Panichpapiboon and W. Pattara-Atikom, "A review of information dissemination protocols for vehicular ad hoc networks", Communications Surveys \& Tutorials, IEEE, 14.3: 784-798, 2012.

[14] L. J. Chen, Y. Y. Chen, K. C. Lan and C. M. Chou, "Localized data dissemination in vehicular sensing networks", Vehicular Networking Conference (VNC), 2009 IEEE. IEEE, 2009.

[15] P. Juang, H. Oki, Y. Wang, M. Martonosi, L. S. Peh and D. Rubenstein, "Energy-efficient computing for wildlife tracking: Design tradeoffs and early experiences with ZebraNet”, ACM Sigplan Notices, 37.10: 96-107, 2002.

[16] J. Capitan, L. Merino, F. Caballero and A. Ollero, "Decentralized Delayed-State Information Filter (DDSIF): A new approach for cooperative decentralized tracking", Robotics and Autonomous Systems 59.6, 376-388, 2011.

[17] Y. Bar-Shalom, R. X. Li and T. Kirubarajan, "Estimation with applications to tracking and navigation: theory algorithms and software", John Wiley and Sons, 2004.

[18] C. Sommer, R German and F. Dressler, "Bidirectionally coupled network and road traffic simulation for improved IVC analysis", Mobile Computing, IEEE Transactions on, 10.1, 3-15, 2011.

[19] A. Varga, "The OMNeT++ discrete event simulation system", Proceedings of the European simulation multiconference (ESM2001), Vol. 9, 2001.

[20] D. Krajzewicz, J. Erdmann, M. Behrisch and L. Bieker, "Recent development and applications of SUMO - simulation of urban mobility", International Journal On Advances in Systems and Measurements, 2012.

[21] G. Gurczik, L. T. Tcheumadjeu, A. Luber, S. Ruppe and M. Junghans, "Floating Observer Information Processing on the Basis of Mobile Bluetooth Data", In Proceedings of the International Conference on Engineering and Applied Sciences Optimization (OPT-i), 2014.

[22] B. J. Kenney, 'Dedicated short-range communications (DSRC) standards in the United States", Proceedings of the IEEE, 99.7, 1162-1182, 2011.

[23] R. Jain, "The art of computer systems performance analysis", John Wiley \& Sons, 2008. 